\title{
BMJ Open Understanding families' experiences following a diagnosis of non-syndromic craniosynostosis: a qualitative study
}

\author{
Victoria Kuta, ${ }^{1}$ Lauren Curry, ${ }^{2}$ Daniel McNeely, ${ }^{3}$ Simon Walling, ${ }^{3}$ Jill Chorney, ${ }^{4}$ \\ Michael Bezuhly (D) ${ }^{5}$
}

To cite: Kuta V, Curry L, McNeely D, et al. Understanding families' experiences following a diagnosis of nonsyndromic craniosynostosis: a qualitative study. BMJ Open 2020;10:e033403. doi:10.1136/ bmjopen-2019-033403

- Prepublication history for this paper is available online. To view these files, please visit the journal online (http://dx.doi org/10.1136/bmjopen-2019033403).

Received 08 August 2019 Revised 08 July 2020 Accepted 04 August 2020

Check for updates

(c) Author(s) (or their employer(s)) 2020. Re-use permitted under CC BY-NC. No commercial re-use. See rights and permissions. Published by BMJ.

${ }^{1}$ Division of Otolaryngology, Dalhousie University, Halifax, Nova Scotia, Canada

${ }^{2}$ Division of General Internal Medicine, Dalhousie University, Halifax, Nova Scotia, Canada

${ }^{3}$ Division of Neurosurgery, IWK Health Centre, Dalhousie University, Halifax, Nova Scotia, Canada

${ }^{4}$ Department of Anaesthesia, Pain Management and Perioperative Medicine, IWK Health Centre, Dalhousie University, Halifax, Nova Scotia, Canada

${ }^{5}$ Division of Plastic Surgery, IWK Health Centre, Dalhousie University, Halifax, Nova Scotia, Canada

Correspondence to Dr Michael Bezuhly; mbezuhly@dal.ca

\section{ABSTRACT}

Objectives Craniosynostosis is typically diagnosed and surgically corrected within the first year of life. The diagnosis and surgical correction of the condition can be a very stressful experience for families. Despite this, there is little research exploring the impact that craniosynostosis has on families, especially in the period immediately following diagnosis and correction. In this study, the authors aimed to qualitatively examine the psychosocial experience of families with a child diagnosed with craniosynostosis.

Design Qualitative study.

Setting Tertiary care paediatric health centre.

Participants Mothers of children newly diagnosed with single-suture, non-syndromic craniosynostosis. Intervention Semistructured interviews regarding parental experience with the initial diagnosis, their decision on corrective surgery for their child, the operative experience, the impact of craniosynostosis on the family and the challenges they encountered throughout their journey.

Primary and secondary outcome measures Thematic analysis, a type of qualitative analysis that provides an in-depth account of participant's experiences, was used to analyse the interview data.

Results 0ver a 4-year period, 12 families meeting eligibility criteria completed the study. Three main themes (six subthemes) emerged from the preoperative interviews: frustration with diagnostic delays (parental intuition and advocacy, hope for improved awareness), understanding what to expect (healthcare supports, interest in connecting with other families) and justifying the need for corrective surgery (influence of the surgeon, struggle with cosmetic indications). Two main themes (four subthemes) were drawn from the postoperative interviews: overcoming fear (the role of healthcare professionals, transition home) and relief (reduction in parental anxiety, cosmetic improvements).

Conclusions Overall, the diagnosis of craniosynostosis has a profound impact on families, leading them to face many struggles throughout their journey. A better understanding of these experiences will help to inform future practice, with a hope to improve this experience for other families moving forward.

\section{INTRODUCTION}

Craniosynostosis, a congenital anomaly involving abnormal fusion of calvarial

\section{Strengths and limitations of this study}

The prospective, qualitative study involving semistructured interviews allowed mothers of children with craniosynostosis to richly describe their journey from the point of diagnosis through to the postoperative period.

- This study examined both major preoperative and postoperative themes within the same cohort, allowing for identification of how maternal concerns change over the course of the treatment of their child.

- Given the small, homogeneous group of participants included, it is unclear whether the results accurately represent the experience of other populations.

sutures, affects 1 in every 2000-3000 live births. ${ }^{1-4}$ It is traditionally classified as either syndromic or non-syndromic. Non-syndromic synostosis is not associated with other dysmorphisms outside the abnormal craniofacial morphology, and typically involves only a single suture. The most common subtypes include sagittal, metopic, unicoronal, bicoronal and lambdoidal. Non-syndromic craniosynostosis is classically treated with corrective surgery within the first year of life, with inconclusive evidence that earlier intervention may be beneficial for certain subtypes. ${ }^{5}$

While still controversial, there is increasing evidence that non-syndromic craniosynostosis may be associated with long-term neurodevelopmental deficits, including difficulties with visuospatial skills, memory, speech and language and learning disorders. ${ }^{5}$ Further studies have suggested that these impairments will persist and cannot be prevented with corrective surgery. ${ }^{6-8}$ Despite this inconclusive evidence, most parents opt for corrective surgery to remodel the skull and allow for normal head growth in their child.

Although the impact of non-syndromic craniosynostosis on neurocognitive development remains in question, children with this 
congenital anomaly may be faced with social and psychological barriers that negatively impact their self-esteem and social function owing to their abnormal appearance. ${ }^{910}$ While many reports document the psychosocial aspects of craniosynostosis from the perspective of the patient, they do not detail the experience of the family. Because corrective surgery is typically performed when patients are infants, parents are responsible for making proxy decisions and are actively involved in patient care. Thus, to obtain a true understanding of early experiences with craniosynostosis, it is important to expand our scope and study not the just the patient, but the family.

Previous studies that attempted to quantify parental stress levels found no difference in the level of stress experienced by parents of children with and without singlesuture craniosynostosis before corrective surgery. ${ }^{11-15}$ Other studies have examined parental satisfaction with their child's postoperative results, with high satisfaction with surgical outcomes generally reported..$^{15-17}$

The aim of the current study is to provide an in-depth qualitative description of families' experiences with craniosynostosis. By adopting a qualitative approach involving semistructured interviews, we allowed families to richly describe their journey and freely communicate personally meaningful topics. This study prospectively explored the experience of families beginning at the time of diagnosis and continuing to the postoperative period. We aim to use our findings to inform future research and practice, with the hope of improving the overall experience for families facing this diagnosis in the future.

\section{METHODS}

Thematic analysis was used as the qualitative methodology. Thematic analysis is a method for identifying, analysing and reporting, in detail, patterns within participants' experiences of an event. ${ }^{18}$ The role of the researcher in the interpretation is also recognised.$^{20}$ Rather than testing a specific hypothesis, this method allows for flexible exploration of a topic in a small, homogeneous sample of respondents for whom the topic is particularly relevant. Thematic analysis informed both the data collection and the reporting for the current study. The interviewer received thematic analysis training under one of the senior authors (J.C) using previously described methodology. ${ }^{19}$ The interviewer was not directly involved in the management of patients.

\section{Patient and public involvement}

Neither patients nor the public were involved in the study design. The research question was developed based on the comments expressed to the corresponding author by several families with a diagnosis of craniosynostosis regarding the need for timelier referral to the craniofacial programme and a need for additional teaching resources to primary care providers on the diagnosis. While these families were the impetus for the research question, they did not directly participate in the design
Table 1 Participant demographics $(n=12)$

\begin{tabular}{ll}
\hline Variable & N (\%) \\
\hline Age, years & $\begin{array}{l}32.4 \pm 6.3 \\
\text { (range 19-42) }\end{array}$ \\
\hline Relationship to patient & $12(100)$ \\
\hline Mother & $0(0)$ \\
\hline Father & \\
\hline Location & $9(75)$ \\
\hline Home address $<50 \mathrm{~km}$ from hospital & $3(25)$ \\
\hline Home address $>50 \mathrm{~km}$ from hospital & \\
\hline Sex of child & $10(83.3)$ \\
\hline Male & $2(16.6)$ \\
\hline Female & \\
\hline Craniosynostosis type & $6(50.0)$ \\
\hline Sagittal & $4(33.3)$ \\
\hline Coronal & $2(16.7)$ \\
\hline
\end{tabular}

or conduct of the study. As stated below, participants were given the opportunity to review a summary of the themes and provide feedback following data analysis.

\section{Study sample}

Institutional research ethics approval was obtained for this study from the IWK Health Centre Research Ethics Board. All families presenting to the Izaak Walton Killam (IWK) Health Centre with a child who received a new diagnosis of non-syndromic craniosynostosis were eligible for this study. These families were identified prospectively by participating surgeons between 15 February 2016 and 15 February 2018. Eligible families were informed of the study by one of the participating surgeons during their initial consult, after receiving a diagnosis. Families were then consented to have their contact information provided to the principle investigator of the study. Twelve eligible families were identified and enrolled into the study over the enrolment period. This sample size is typical for thematic analysis studies to reach thematic saturation. ${ }^{19}$ Participant demographics are presented in table 1. All participants were mothers and were interviewed individually.

\section{Data collection}

Participants completed two phone interviews. The first interview was completed within a month of receiving the initial diagnosis. The second interview was completed 3 months postoperatively, or 3 months after the initial interview if the family decided not to proceed with surgery. All interviews were completed by the first author. Verbal consent was obtained over the phone before initiating the interviews. Interviews were semistructured using an interview guide based on the team's clinical experience and a scoping literature review. The initial interview guide (see online supplemental file) contained questions 
on the diagnostic experience as well as the decision on corrective surgery. The second interview guide investigated the surgical experience and the recovery period.

\section{Analysis method}

Interviews were recorded and transcribed verbatim by a professional transcriptionist with subsequent deidentification. Transcripts were divided into preoperative and postoperative categories. Initial coding was completed by the first author using thematic analysis methods. Transcripts were reviewed multiple times to assign codes to the main topics discussed by the participant. The codes identified in earlier transcripts informed the coding of later transcripts. New codes identified in later transcripts prompted earlier transcripts to be reviewed again to determine if these codes were also present in these. The organisation of themes followed an iterative process aiming to identify the meaning behind participants' statements rather than solely the prevalence of topics discussed. Themes were then clustered, allowing for superordinate themes to be generated based on subsumption and abstraction techniques.

To ensure rigour, theme development was reviewed and discussed between the first and senior authors to confirm that the interpretations accurately represented the transcript data. A second author reviewed the transcripts independently to assess for representativeness. Member checking was also performed, where participants were given the opportunity to review a summary of the themes and provide feedback.

\section{RESULTS}

Themes were organised into preoperative and postoperative categories. Three main themes emerged from the preoperative interviews: frustration with diagnostic delays, understanding what to expect and justifying the need for corrective surgery. Two main themes emerged from the postoperative interviews: overcoming fear and relief. Representative quotes are included throughout the text.

\section{Frustration with diagnostic delays}

Most participants expressed some frustration around diagnostic delays, excepting two participants whose child was born at the tertiary care hospital and received a diagnosis immediately post partum. Two subthemes emerged: parental intuition/advocacy and hope for improved awareness.

\section{Parental intuition and advocacy}

Ten families noticed the abnormal shape of their child's head at birth and expressed concerns (Participant 12-'I knew something was wrong, but I couldn't prove it'). They were frequently offered the explanation that it was a result of the birthing process and were told it would resolve spontaneously (Participant 2-'The day he was born at the hospital we started noticing that one of his eyes would not open, and his nose was crooked a bit and the opening in one of his nostrils was very narrow. We were told it was because of what they call a traumatic birth, and it would fix as he grows.')

Over time, when no aesthetic improvement was observed, families began seeking medical advice. One family requested an X-ray; however, the diagnosis of craniosynostosis was missed. Other families resorted to taking their child to the emergency room or requesting a referral to a paediatrician after feeling their concerns were not adequately addressed by their family physician. One family expressed feelings of guilt around not pushing for the referral to a specialist earlier (Participant 2-'I started doing my own research online and that's when I realised something should have been done when he was younger. I was a bit frustrated with my doctor. I felt like I should have pushed for it sooner when he was younger').

\section{Hope for improved awareness}

Overall, families describe a lack of awareness among community family physicians around craniosynostosis. One mother explains her surprise that the craniosynostosis wasn't picked up by her family physician despite regular exams (Participant 5-'At every doctor's appointment they are always doing measurements of his head and looking for his soft spot'). Another mother describes her own physical findings that she felt were discounted (Participant 6-'I also noticed a ridge along the top of his skull that I brought up to my GP and he kind of passed if off as not a big deal'). When asked how their overall experience could be improved, many parents suggested efforts to increase craniosynostosis awareness to allow for earlier detection (Participant 10-'Being able to have more education for family doctors, nurse practitioners, that sort of thing, around what is normal and what's not normal'; Participant 2-'I think it's something they should be more educated on.')

\section{Understanding what to expect}

Participants described the importance of being informed on what to expect and how this helped them to feel more comfortable during the whole experience. Two subthemes emerged: healthcare supports, and interest in connecting with other families.

\section{Healthcare supports}

Participants described feeling overwhelmed during their initial consultation, and most were unaware that surgery would be recommended for their child. Many had come mentally prepared with questions, but were then unable to recall these during the consult (Participant 1-'So when he said 'do you have any questions' I was like 'no' because I was just trying to take it all in'). Other families chose to write down their questions ahead of time, which proved to be a more successful strategy. One participant commented on too many learners being present in the room-a comment that nursing staff later agreed with. This added to the overwhelming nature of the consult 
and hindered this participant's ability to express themselves. All participants described receiving verbal information; however, many suggested that additional written resources could have been provided for review once they have had time to process things (Participant 10-'So I would say having a cheat sheet of something, where it's already written down that you leave with. Because in the moment, you're listening and not thinking of writing it down yourself'). Skull models used during the consult were helpful for participant education. As many participants were doing their own research, they requested references to reputable resources for further information. Additionally, participants appreciated having access to a specialised nurse after the consultation with the surgeons who they could email or call with additional questions. All participants spoke very highly of this support system and felt that it significantly reduced their anxiety (Participant 10-'It was so helpful to know that if we did [have questions], we had a way to get a hold of [the clinic nurse]').

\section{Interest in connecting with other families}

While all of the participants felt their consultation visits were informative, they expressed a strong interest in connecting with other families who have been through a similar experience (Participant 8-'The doctor told me what I could expect, what I'm going to see after the surgery and all this, but hearing it from a parent's perspective is a whole different story'). Participants felt it was important to hear other local success stories and mentioned that they would like access to preoperative and postoperative photos from other families (Participant 7-'As a mom and dad you really need to see that other children have risen through it'). Many participants reached out to other families through craniosynostosis support groups on social media platforms. They described the support and hope provided through these online chats (Participant 10-'Those connections are important, I think, just to see that there are other people who are going through it and have made it through to the other side'). While most participants thought these types of communication would be helpful, one participant describes her emotional struggle after meeting with a family who experienced complications (Participant 12-'I was scared. I'm even more scared now than I was then, because now that we're in support groups and see what's going to happen, we are scared about the surgery. It's always hard when you have a small sample size too. It can make things look like they are in different proportions than they are').

\section{Justifying the need for corrective surgery}

Participants describe the difficult process of reaching a decision on corrective surgery for their child. Two subthemes emerged: influence of the surgeon and struggle with cosmetic indications.

\section{Influence of the surgeon}

All participants decided to proceed with corrective surgery for their child. This decision was reached during the initial consultation. The families described the importance of the information they received during this surgical visit and stated there were no outside influences factored into their decision. This speaks to the magnitude of the influence held by the surgeons. Many participants describe their positive relationship with the surgeons, and how it gave them confidence to consent to surgery during the first visit (Participant 7-'I feel really confident with the doctors, I feel good with them, which I definitely think is a part of it'). Both bedside manner and the communication style of the surgeons were noted to help participants feel more comfortable with their decision on surgery (Participant 11-'They talk to you like you're a human being. They talk to you in a fashion that, you know, we know what you're actually telling us. But it's easing my mind that we have such a great team'). Participants also appreciated surgeons speaking in lay terms during the consultation and consenting processes. Other families focused primarily on the evidence and risks communicated by the surgeon (Participant 4-'When he told us there are $10 \%-15 \%$ that have pressure build-up in their brain and it can affect development and also his vision, [...] I don't think we had to think very long to decide that we do not want to take that risk if we can definitely prevent it by doing a surgery'). While many families deliberated on this difficult decision, some families describe the feeling of not having a choice, that surgery was the only option (Participant 1-'He was talking about how the shape of his head would just continue to grow this way, and his brain would be squeezed and there would be pressure. But yeah, I felt like this was our only option').

\section{Struggle with cosmetic indications}

The participants discussed their struggle weighing the importance of cosmetic indications, with most families stating that the decision would be much more difficult if the surgery was for aesthetic purposes alone (Participant 4-'If it was just cosmetic it definitely would have taken us more time to think about it.'). While most families identified potential neurological risks as their primary motivator, it seems that aesthetic concerns were still present, even if not directly vocalised (Participant 11-'So we know that it's not a decision that we're being selfish and trying to fix her look. It needs to be completed'). Other families were more direct in voicing their cosmetic concerns and were worried about potential psychosocial difficulties later in life, especially after learning about the potentially progressive nature of the condition. This included concerns around future bullying, depression, and the even the risk of suicide if surgery was not performed (Participant 8-'No child should grow up and develop that head shape'; Participant 12-'When she was first diagnosed, I would have said no, but now, the asymmetry is so much that it wouldn't be fair to her not to repair it. She would always look very different from other children'; Participant 10-'If we don't do the surgery, he's going to hate us later in life because we didn't fix this. He would probably be teased and picked on'). One family 
related their cosmetic concerns to the sex of their child, describing the gender-biased aesthetic standard they have experienced in society (Participant 12-'[My husband] keeps saying specifically because she's a girl, and we live in a society where what a girl looks like is important').

\section{Overcoming fear}

The participants describe fear at various stages of their journey and shared what helped them cope with this emotion. Two subthemes emerged: the role of healthcare professionals and the transition home.

\section{The role of healthcare professionals}

Participants discussed at length how healthcare providers helped reduce their fear and anxiety while in hospital. First, although parents found it very difficult to hand over their child for surgery, the were comforted by regular updates throughout the procedure (Participant 4-'You're just waiting for that nurse to come and give us the news that everything is going well, and like it's supposed to. And she did, every time. That was great'). There was only one family who did not receive regular updates throughout the operation. This participant describes feeling extremely nervous in the waiting room after not being informed about a delay in the surgical start time (Participant 5-'I would have liked to know that they started later than think something bad happened'). Overall, regardless of the stage in their journey, parents described feeling much calmer when they were kept informed. In addition, families commented on the importance of empathy in healthcare. For example, one participant (Participant 10) spoke of the impact that small gestures can have on a family during a difficult time: 'They brought us out the bag of his hair. One of them had written on it 'baby's first haircut'. It let you know that they care about your child, that they see that it's not just another patient.'

\section{Transitioning home}

Most participants were very surprised with the short recovery time after discharge home (Participant 5-'You don't think they are going to recover that quick [...], but within 2 or 3 days they're their normal self'). This introduced a new fear for parents. Many families described difficulty allowing their child to return to regular activities out of fear they would hurt themselves (Participant 8-'We're still really scared, like if he falls and bangs his head or something, we're like 'Ooohh!'). These concerns were heightened if the child had young siblings (Participant 1-'And even now, it's hard, because [my other children] are so young, and he still has the soft spot on his head, but they don't understand'). When asked what helped ease their transition home, families stated that were very grateful to be given contact information to reach their healthcare team with questions after discharge. They felt comfortable emailing or calling members of the team with postoperative questions. Ultimately, the ongoing support for parents helped to reduce feelings of fear and anxiety after discharge.

\section{Relief}

All 12 participants expressed a sense of relief postoperatively, feeling confident they had made the right decision regarding corrective surgery. Two subthemes emerged: reduction in parental anxiety and cosmetic improvements.

\section{Reduction in parental anxiety}

Participants described significant anxiety leading up to the operation, despite feeling very well informed. Many families feared that they would regret their decision regarding corrective surgery and felt a substantial amount of pressure to make the right choice (Participant 7-'My fear was that he would be changed for the worse and that we would forever regret the decision to do it'). All participants felt their anxiety subside post-operatively after a successful operation.

Parents also described significant anxiety around the potential for neurological deficits associated with craniosynostosis, worrying that irreversible effects would occur before surgery (Participant 1-'I was always making sure he could focus on me, and if he couldn't focus on me I'd think 'oh no, is he going blind"). Postoperatively, participants no longer worried about neurological deficits, and felt they were no longer anxious about their child meeting developmental milestones. Many families also described positive behavioural changes in their child that they attributed to the surgery (Participant 7-'He is happier and a little more relaxed. He is able to play more'; Participant 12-'She was almost, I would say, mute leading up to surgery. Within a week of surgery she started making sounds and now, 3 months later, has a full vocabulary').

\section{Cosmetic improvements}

Although most families claimed neurological deficits were their primary motivation for surgery, the cosmetic improvements were heavily commented on in the postoperative interviews (Participant 3-'The best part would be how he looked after surgery. Like 3 weeks after, how good he looked. He looked like a total different baby'). Participants expressed relief with the aesthetic success of the operation (Participant 4-'It did really change the way that his face and features look. It wasn't the main reason for us to do the surgery, but it was definitely, like, "oh wow!"). One mother commented on the practical aspect of her child's new head shape (Participant 6-'I appreciate being able to put a hat on him now'). Another reflected on the progressive nature of craniosynostosis, describing what she felt her child would have looked like now without the operation (Participant 8-'If we never would have done that surgery $[\ldots]$, his head would be so much like a football right now'). Overall, parents seemed very satisfied that their child would no longer stand out due to a cranial deformity (Participant 6-'He looks like a completely normal 8-month old now, besides the really faint scarring'). 


\section{DISCUSSION}

The diagnosis and treatment of craniosynostosis has a significant impact on families. This qualitative analysis provides a rich description of families' experiences with craniosynostosis, from the point of diagnosis through to the period of surgical recovery.

In the preoperative interviews, most families described frustration around diagnostic delay, acknowledging the importance of advocating for their child and their hope for improved craniosynostosis awareness in community practice. They stressed the importance of knowing what to expect, and the value in both healthcare supports and making connections with other families. They also discussed the struggle to decide on corrective surgery, acknowledging the influence of the surgeon and their difficulties weighing functional and cosmetic indications.

In the postoperative interviews, families discussed their journey of overcoming their fear. They highlighted the contribution of healthcare professionals and emphasised the challenges of transitioning home. There was also a very different tone to the second round of interviews, one of relief. All families were happy with their decision to proceed with corrective surgery, and felt their anxiety was reduced in the postoperative period. They also commented on their satisfaction with the cosmetic improvement.

Overall, the preoperative themes centred around feelings of uncertainty and illustrate the struggle families experience in the early stages of this process. Parents were often left questioning their decisions and wondering if they were making the right choice for their child. In this period of uncertainty, parents tended to place significant weight on the opinion of their child's surgeon and draw confidence from this interaction. In contrast, the postoperative period was characterised by themes illustrating strength and independence. Although uncertainty still existed throughout the recovery period, parents appeared well equipped to handle these challenges as a family unit, needing less reassurance from healthcare professionals. All parents included in this study described a positive change in their child after surgery. Parents appeared to draw strength from the fact that their decision to proceed with surgery was what led to this positive outcome. Also, while parents acted as advocates for their own children in the preoperative period, they advocated for the craniosynostosis community at large in the postoperative period, once again illustrating their personal growth and confidence.

For most forms of non-syndromic craniosynostosis the prevention of elevated intracranial pressure and associated neurocognitive deficits is the principal indication for surgery. ${ }^{21}$ Sagittal craniosynostosis may be an exception, as compensatory growth along patent sutures largely prevents elevated intracranial pressure but produces a stigmatising head shape. To this end, aesthetic concerns may be a greater motivation for surgical correction of sagittal craniosynostosis. ${ }^{22}$ A recent health utility outcome study found relatively high utility scores for sagittal craniosynostosis, suggesting that the cosmetic burden of this condition as perceived by the general population is low. ${ }^{23}$ This aligns with our findings, where most participants stated that the cosmetic indications for corrective surgery were secondary to the neurological ones. The observation that concerns regarding cognitive sequelae were the main motivation for corrective surgery underscores the need for ongoing clinical research into functional aspects of craniosynostosis management. Of note, all families decided to proceed with corrective surgery, including those who received a diagnosis of sagittal craniosynostosis. While families may find it difficult to choose a potentially morbid corrective surgery for aesthetic indications alone, it is important to remind parents of the potential psychosocial consequences of living with an uncorrected craniofacial abnormality. ${ }^{24}$ Interestingly, despite the difficulty justifying aesthetic indications preoperatively, the satisfaction with cosmetic improvements was heavily commented on in the postoperative interviews.

Many of the themes developed in our study align with those reported by previous studies examining the experiences of families with children diagnosed with other craniofacial deformities. For example, parents with children diagnosed with cleft lip/palate described their anxiety around surgery and their need for emotional support throughout treatment, for both themselves and their child. ${ }^{25-27}$ Furthermore, families of children diagnosed with craniofacial abnormalities have expressed fears that their child will be bullied and ostracised later in life. ${ }^{2527}$ Previous studies have emphasised the importance of parental support in healthcare, suggesting that the emotional state of caregivers significantly influences the emotional development of children with craniofacial abnormalities. ${ }^{27-29}$ Elevated caregiver stress was found to have long-lasting, negative psychosocial effects on children who received corrective surgery for craniofacial abnormalities and was also associated with increased levels of anxiety and depression among patients during childhood. $^{30}$ While it is conceivable that surgical 'normalisation' of appearance may have a salutary effect on parent-child interactions, the evidence for this is conflicting, with some demonstrating that mothers may be more protective of children with craniofacial differences thus leading to stronger attachment. ${ }^{31-33}$ By better understanding the experience of craniosynostosis by families, supports can be appropriately tailored to address current areas of concern and improve the overall experience.

While many of our themes supported previous research, frustration with diagnostic delays was a key theme in our study and has not previously been reported for craniosynostosis. While there are currently no guidelines outlining the optimal age for surgical correction of craniosynostosis, much research has focused on this issue. A systematic review by Mandela et al found no conclusive evidence that earlier surgery may be beneficial to patients with sagittal synostosis, for example. There is no evidence that later surgery is beneficial for any of the craniosynostosis 
subtypes. ${ }^{5}$ This speaks to the importance of early detection, as the age of diagnosis will affect when and what type of surgery is offered. One family in our study received the diagnosis of craniosynostosis early in the postpartum period. Due to the young age at detection, the child was eligible for less invasive endoscopic correction and helmet therapy. This option would not have been offered had the family experienced a diagnostic delay like that experienced by most families in our series.

In addition to improving craniosynostosis awareness, families also suggested that it would have been helpful to have received printed material during the initial consult to complement the information that was provided verbally. They expressed interest in receiving written pamphlets as well as a list of reliable and recommended internet sources where they could review the information further. The provision of these decision aids has previously been found to increase both comprehension and risk recall. ${ }^{34}$ In addition to improving informed consent, these interventions may increase overall satisfaction with the decision-making process. ${ }^{35}$ This is especially relevant to the craniosynostosis patient population, as parents described significant anxiety associated with therapeutic decision making.

This study is not without limitations. First, given the small, homogeneous group of participants included, it is unclear whether our results accurately represent the experience of other populations. All 12 families elected to have the patient's mother complete the interviews rather than the father, regardless of marital status. While this may reflect the traditional distribution of caregiver responsibilities, it prevents us from identifying potential meaningful gender differences in the family experience of craniosynostosis. For example, parental stress has previously been reported to be higher in mothers with children diagnosed with single suture craniosynostosis when compared with their paternal counterparts. ${ }^{36}$ Second, because our study design assigned recruitment responsibilities to the participating surgeons, the surgeons were not blinded to which families were enrolled. Although participants were ensured anonymity, it is unclear whether this influenced the interviews, potentially making participants more reluctant to identify points of dissatisfaction around their interactions with the surgeons. Despite these limitations, our study offers important insights for physicians caring for children with craniosynostosis and helps healthcare providers better understand the needs of families during the preoperative, perioperative and postoperative periods.

This study also suggests future avenues of research and development. Despite the fear expressed by parents in the preoperative period, all families were ultimately pleased with their decision to proceed with corrective surgery. Future studies aim to explore the opinion of the patients themselves, and their views on their parents' decision regarding surgical correction of their craniosynostosis. Additionally, our findings speak to the importance of lifelong learning in the medical field and identify the need for additional craniosynostosis teaching among general practitioners to allow for earlier detection in the community.

\section{CONCLUSION}

The diagnosis of craniosynostosis has a significant impact on families. This study offers a detailed look into the experiences of families from the point of diagnosis through to the postoperative recovery and transition home. Participants provide rich descriptions of their frustrations, accomplishments, supports and their suggestions for improvement. A better understanding of this experience will identify where further supports are needed and inform future practice, with the goal of improving the overall experience for other families moving forward.

Contributors VK: Literature review, ethics submission, interviews, primary thematic analysis, manuscript preparation and submission. LC: Secondary reviewer of transcripts and themes. DM: Patient recruitment, surgery, manuscript preparation. SW: Patient recruitment, surgery, manuscript preparation. JC: Ethics submission, study design, manuscript preparation. MB: Primary supervisor, ethics submission, study design, patient recruitment, surgery, manuscript preparation and submission.

Funding The authors have not declared a specific grant for this research from any funding agency in the public, commercial or not-for-profit sectors.

Competing interests None declared.

Patient and public involvement Patients and/or the public were not involved in the design, or conduct, or reporting, or dissemination plans of this research.

Patient consent for publication Not required.

Provenance and peer review Not commissioned; externally peer reviewed.

Data availability statement Data are available in a public, open access repository. Extra data can be accessed via the Dryad data repository at https://datadryad.org/ stash/dataset/doi:10.5061/dryad.fr9305r.

Open access This is an open access article distributed in accordance with the Creative Commons Attribution Non Commercial (CC BY-NC 4.0) license, which permits others to distribute, remix, adapt, build upon this work non-commercially, and license their derivative works on different terms, provided the original work is properly cited, appropriate credit is given, any changes made indicated, and the use is non-commercial. See: http://creativecommons.org/licenses/by-nc/4.0/.

ORCID iD

Michael Bezuhly http://orcid.org/0000-0002-7356-5147

\section{REFERENCES}

1 Zhang G, Tan H, Qian X, et al. A systematic approach to predicting spring force for sagittal craniosynostosis surgery. J Craniofac Surg 2016;27:636-43.

2 Cornelissen M, Ottelander BD, Rizopoulos D, et al. Increase of prevalence of craniosynostosis. J Craniomaxillofac Surg 2016;pii: S1010-5182:30130-5.

3 Lajeunie E, Le Merrer M, Bonaïti-Pellie C, et al. Genetic study of nonsyndromic coronal craniosynostosis. Am J Med Genet 1995;55:500-4.

4 Garza RM, Khosla RK. Nonsyndromic craniosynostosis. Semin Plast Surg 2012;26:053-63.

5 Mandela R, Bellew M, Chumas P, et al. Impact of surgery timing for craniosynostosis on neurodevelopmental outcomes: a systematic review. J Neurosurg Pediatr 2019;23:442-54.

6 Kapp-Simon KA, Speltz ML, Cunningham ML, et al. Neurodevelopment of children with single suture craniosynostosis: a review. Childs Nerv Syst 2007;23:269-81.

7 Knight SJ, Anderson VA, Spencer-Smith MM, et al. Neurodevelopmental outcomes in infants and children with singlesuture craniosynostosis: a systematic review. Dev Neuropsychol 2014;39:159-86. 
8 Speltz ML, Kapp-Simon KA, Cunningham M, et al. Single-suture craniosynostosis: a review of neurobehavioral research and theory. $J$ Pediatr Psychol 2004;29:651-68.

9 Speltz ML, Collett BR, Wallace ER, et al. Behavioral adjustment of school-age children with and without single-suture craniosynostosis. Plast Reconstr Surg 2016;138:435-45.

10 Singh VP, Moss TP. Psychological impact of visible differences in patients with congenital craniofacial anomalies. Prog Orthod 2015;16:5

11 Rosenberg JM, Kapp-Simon KA, Starr JR, et al. Mothers' and fathers' reports of stress in families of infants with and without single-suture craniosynostosis. Cleft Palate Craniofac J 2011;48:509-18.

12 Cloonan YK, Collett B, Speltz ML, et al. Psychosocial outcomes in children with and without non-syndromic craniosynostosis: findings from two studies. Cleft Palate Craniofac J 2013;50:406-13.

13 Gray KE, Cradock MM, Kapp-Simon KA, et al. Longitudinal analysis of parenting stress in mothers and fathers of infants with and without single-suture craniosynostosis. Cleft Palate Craniofac $J$ 2015;52:3-11.

14 Kapp-Simon KA, Leroux B, Cunningham M, et al. Multisite study of infants with single-suture craniosynostosis: preliminary report of presurgery development. Cleft Palate Craniofac J 2005:42:377-84.

15 Lim J, Davis A, Tang AR, et al. Caregiver stress in children with craniosynostosis: a systematic literature review. Childs Nerv Syst 2019;35:217-25.

16 Wong-Gibbons DL, Kancherla V, Romitti PA, et al. Maternal reports of satisfaction with care and outcomes for children with craniosynostosis. J Craniofac Surg 2009;20:138-42.

17 Kluba S, Rohleder S, Wolff M, et al. Parental perception of treatment and medical care in children with craniosynostosis. Int J Oral Maxillofac Surg 2016;45:1341-6.

18 Attride-Stirling J. Thematic networks: an analytic tool for qualitative research. Qual Res 2001:1:385-405.

19 Braun V, Clarke V. Using thematic analysis. Qual Res Psychol 2006;3:77-101.

20 Taylor GW, Ussher JM. Making sense of S\&M: a discourse analytic account. Sexualities 2002;4:293-314.

21 Tamburrini G, Caldarelli M, Massimi L, et al. Intracranial pressure monitoring in children with single suture and complex craniosynostosis: a review. Childs Nerv Syst 2005;21:913-21.
22 Mathijssen IMJ. Guideline for care of patients with the diagnoses of craniosynostosis: working group on craniosynostosis. J Craniofac Surg 2015;26:1735-807.

23 Kuta V, McNeely PD, Walling S, et al. Sagittal craniosynostosis: a utility outcomes study. J Neurosurg Pediatr 2017;20:113-8.

24 Boltshauser E, Ludwig S, Dietrich F, et al. Sagittal craniosynostosis: cognitive development, behaviour, and quality of life in unoperated children. Neuropediatrics 2003;34:293-300.

25 Klein T, Pope AW, Getahun E, et al. Mothers' reflections on raising a child with a craniofacial anomaly. Cleft Palate Craniofac $J$ 2006; $43: 590-7$

26 Johansson B, Ringsberg KC. Parents' experiences of having a child with cleft lip and palate. J Adv Nurs 2004;47:165-73.

27 Nelson P, Glenny A-M, Kirk S, et al. Parents' experiences of caring for a child with a cleft lip and/or palate: a review of the literature. Child Care Health Dev 2012;38:6-20.

28 Coulter ML, Scheuerle J, Laude M, et al. Psychological aspects of parents of children with craniofacial anomalies. J Craniofac Surg 1991;2:9-17.

29 Campis LB, DeMaso DR, Twente AW. The role of maternal factors in the adaptation of children with craniofacial disfigurement. Cleft Palate Craniofac J 1995;32:55-61.

30 Pillemer FG, Cook KV. The psychosocial adjustment of pediatric craniofacial patients after surgery. Cleft Palate J 1989;26:201-7.

31 Despars J, Peter C, Borghini A, et al. Impact of a cleft lip and/or palate on maternal stress and attachment representations. Cleft Palate Craniofac J 2011;48:419-24.

32 Coy K, Speltz ML, Jones K. Facial appearance and attachment in infants with orofacial clefts: a replication. Cleft Palate Craniofac $J$ 2002;39:66-72

33 Maris CL, Endriga MC, Speltz ML, et al. Are infants with orofacial clefts at risk for insecure mother-child attachments? Cleft Palate Craniofac J 2000;37:257-65.

34 Papsin E, Haworth R, Chorney JM, et al. Pediatric otoplasty and informed consent: do information handouts improve parental risk recall? Int J Pediatr Otorhinolaryngol 2014;78:2258-61.

35 Leatherman S, Warrick L. Effectiveness of decision AIDS: a review of the evidence. Med Care Res Rev 2008;65:79S-116.

36 Rosenberg JM, Kapp-Simon KA, Starr JR, et al. Mothers' and fathers' reports of stress in families of infants with and without singlesuture craniosynostosis. Cleft Palate Craniofac J 2011;48:509-18. 